\title{
Congestion Management in Deregulated Power System by Locating Series FACTS Devices
}

\author{
L.Rajalakshmi \\ Associate Professor/EEE \\ KLN College of Information \\ Technology \\ Sivagangai, India
}

\author{
M.V.Suganyadevi \\ Assistant Professor/EEE \\ KLN College of Engineering \\ Sivagangai, India
}

\author{
S.Parameswari \\ Assistant Professor/EEE \\ KLN College of Information \\ Technology \\ Sivagangai, India
}

\begin{abstract}
Congestion management is one of the technical challenges in Power system deregulation. In deregulated electricity market transmission congestion occurs when there is insufficient transmission capacity to simultaneously accommodate all constraints for transmission of a line. Flexible Alternative Current Transmission System (FACTS) devices can be an alternative to reduce the flows in heavily loaded lines, resulting in an increased loadability, low system loss, improved stability of the network, reduced cost of production and fulfilled contractual requirement by controlling the power flow in the network. A method to determine the optimal location of FACTS has been suggested based on real power Performance Index and reduction of total system VAR power losses. The simulation results were successfully tested on IEEE 14 bus system.
\end{abstract}

\section{Keywords}

congestion management, FACTS devices, deregulated power system, performance index.

\section{INTRODUCTION}

Transmission lines are often driven close to or even beyond their thermal limits in order to satisfy the increased electric power consumption and trades due to increase of the unplanned power exchanges. If the exchanges were not controlled, some lines located on particular paths may become overloaded, this phenomenon is called congestion. The management of congestion is somewhat more complex in competitive power markets and leads to several disputes. Congestion may be alleviated through various ways. Among the technical solutions, we have system redispatch, system reconfiguration, outaging of congested lines, operation of FACTS devices and operation of transformer tap changers. A number of studies have devoted to the congestion management problems using generation redispatch, security constrained optimal power flow, and load curtailment combined with redispatch [1]-[4].

The issue of transmission congestion is more pronounced in deregulated and competitive markets and it needs a special treatment. In this environment, independent system operator (ISO) has to relieve the congestion, so that the system is maintained in secure state. To relieve the congestion ISO can use mainly two types of techniques which are as follows:
A. Cost free means:
- $\quad$ Out-ageing of congested lines
- Operation of transformer taps/phase shifters
- Operation of FACTS devices particularly series devices
B. Non-Cost free means:

- Re-dispatching the generation amounts. By using this method, some generators back down while others increase their output. The effect of re-dispatching is that generators no longer operate at equal incremental costs.

- Curtailment of loads and the exercise of load interruption options.

Among the above two main techniques cost free means do have advantages such as not touching economical matters, so GENCO and DISCO will not be involved. Hence, FACTS devices are utilized as one of such technology which can reduce the transmission congestion and leads to better using of the existing grid infrastructure. Besides, using FACTS devices gives more opportunity to ISO. Various issues associated with the usage of FACTS devices are their optimal location and appropriate size, setting, cost, and modeling.

FACTS devices, especially series FACTS devices like TCSC are considered one such technology that reduced the transmission congestion and allows better utilization of the existing grid infrastructure, along with many other benefits. Various issues associated with the use of FACTS devices are proper location, appropriate size and setting, cost, modeling, and controller interactions. This paper deals with the location aspect of the series FACTS devices, especially to manage congestion in the deregulated electricity markets.

The location of FACTS devices can be based on static or dynamic performance of the system. In [5], an overload sensitivity factor (power flow index) is used for optimal location of series FACTS devices for static congestion management. A loss sensitivity factor method is used in [6] to determine the suitable location for FACTS devices.

This paper presents the comparative analysis of methodologies based on real power Performance Index and reduction of total system VAR power losses for proper location for congestion management in the deregulated electricity markets. In Section 2 static modeling of FACTS devices and formulation is obtained. In Section 3 the optimal location is based on the minimizing the production and device cost. In Section 4 the results and discussion were present. Also at the end, line outage as a contingency analysis has been discussed.

\section{STATIC MODELLING OF FACTS DEVICES AND FORMULATION}

For static application like congestion management FACTS devices can be modeled as Power Injection Model [7]. The injection model describes the FACTS devices as a device that injects a certain amount of active and reactive power to a node, so that the FACTS devices are presented as PQ elements. During steady state operation, TCSC can be considered as an additional reactance $-j x c$, The value of $x c$ is adjusted according to control scheme specified. Fig. 1(a) shows a model of transmission line 
with one TCSC which is connected between bus- $i$ and bus- $j$. The line flow change is due to series capacitance which is represented as line without series capacitance with power injected at the receiving and sending ends of the line as shown in Fig. 1(b).

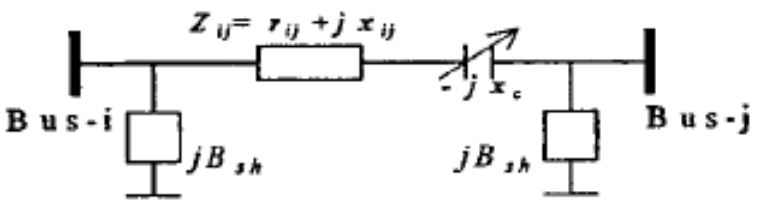

Fig.1 (a) TCSC model

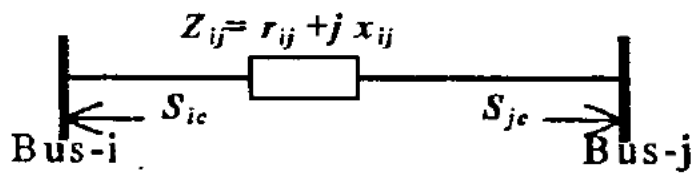

Fig.1 (b) Injection model of TCSC

The real power injections at bus- $i$ (Pic) and bus- $j($ Pjc $)$ are given by [8]:

$P_{i c}=V_{i}^{2} \Delta G_{i j}-V_{i} V_{j}\left[\Delta G_{i j} \cos \delta_{i j}+\Delta B_{i j} \sin \delta_{i j}\right]$
$P_{j c}=V_{j}^{2} \Delta G_{i j}-V_{i} V_{j}\left[\Delta G_{i j} \cos \delta_{i j}-\Delta B_{i j} \sin \delta_{i j}\right]$

Similarly, the reactive power injections at bus- $i$ (Qic ) and bus$j(Q j c)$ can be expressed as:

$$
\begin{aligned}
& Q_{i c}=-V_{i}^{2} \Delta B_{i j}-V_{i} V_{j}\left[\Delta G_{i j} \sin \delta_{i j}-\Delta B_{i j} \cos \delta_{i j}\right] \\
& P_{j c}=-V_{j}^{2} \Delta B_{i j}-V_{i} V_{j}\left[\Delta G_{i j} \sin \delta_{i j}+\Delta B_{i j} \cos \delta_{i j}\right]
\end{aligned}
$$

Where

$$
\begin{aligned}
\Delta G_{i j} & =\frac{x_{c} r_{i j}\left(x_{c}-2 x_{i j}\right)}{\left(r^{2}{ }_{i j}+x_{i j}^{2}\right)\left(r^{2}{ }_{i j}+\left(x_{i j}-x_{c}\right)^{2}\right)} \\
\Delta B_{i j} & =\frac{-x_{c}\left(r^{2}{ }_{i j}-x^{2}{ }_{i j}+x_{c} x_{i j}\right)}{\left(r^{2}{ }_{i j}+x^{2}{ }_{i j}\right)\left(r^{2}{ }_{i j}+\left(x_{i j}-x_{c}\right)^{2}\right)}
\end{aligned}
$$

This model of TCSC is used to properly modify the parameters of transmission lines with TCSC for optimal location.

Due to high cost of FACTS devices, it is necessary to use cost benefit analysis to analyze whether new FACTS device is cost effective among several candidate locations where they actually installed. The TCSC cost in line-k is given by [9]

$$
C_{T C S C}(k)=c \cdot x_{c}(k) \cdot P_{L}^{2} \cdot \text { Base_Power }
$$

where $\mathrm{c}$ is the unit investment cost of FACTS, $\mathrm{xc}(\mathrm{k})$ is the series capacitive reactance and PL is the power flow in line-k. The objective function for placement of TCSC will be

$$
\min _{P i} \sum_{i} C_{i}\left(P_{i}\right)+C_{T C S C}
$$

\section{OPTIMAL LOCATION OF FACTS DEVICES}

A. DC Power Flow Sensitivity Factors:

By definition, the DC power flow sensitivity factors have the following meaning [10]: $d_{l, k}=\frac{\Delta f_{l}}{f_{k}^{0}}$

(6) where $l k d$, is line outage sensitivity factor when monitoring line $l$ after outage of line $k, \Delta f_{l}$ is change in MW flow on line $l$, and $f_{k}^{0}$ is the original flow on line $k$ before its outage. Lines $l$ and $k$ are located between buses i, j and $\mathrm{n}, \mathrm{m}$, respectively. Considering DC power flow formulation:

$\Delta \theta=[X] \Delta P$

where $[\mathrm{X}]$ is reactance matrix of DC power flow, line outage sensitivity factor can be calculated by:

$$
d_{l, k}=\frac{\frac{x_{k}}{x_{l}}\left(X_{i n}-X_{j n}-X_{i m}+X_{j m}\right)}{x_{k}-\left(X_{n n}+X_{m m}-2 X_{n m}\right)}
$$

where $x_{k}$ and $x_{l}$ are reactance of lines $k$ and $l$, respectively, and $X_{m n}$ is $m n^{\text {th }}$ element of DC power flow reactance matrix. Larger Sensitivity factors indicate more dependency.

\section{B. Reduction of Total System VAR Power Loss:}

A method based on the sensitivity of the total system reactive power loss with respect to the control variable of the TCSC. For TCSC placed between buses $i$ and $j$ we consider net line series reactance as a control parameter. Loss sensitivity with respect to control parameter of TCSC placed between buses $i$ and $j$ can be written as:

$$
\begin{aligned}
& a i j=\frac{\partial Q_{L}}{\partial x_{i j}} \\
& =\left[V^{2}{ }_{i}+V^{2}{ }_{j}-2 V_{i} V_{j} \cos \delta_{i j}\right] \frac{r^{2}{ }_{i j}-x_{i j}^{2}}{\left(r^{2}{ }_{i j}+x^{2}{ }_{i j}\right)^{2}}
\end{aligned}
$$

\section{Real Power Flow Performance Index} Sensitivity Indices:

The severity of the system loading under normal and contingency cases can be described by a real power line flow performance index [10], as given below

$$
P I=\sum_{m=1}^{N L} \frac{w_{m}}{2 n}\left(\frac{P L M}{P_{L M}^{\max }}\right)^{2 n}
$$

where $\mathrm{P}_{\mathrm{Lm}}$ is the real power flow and $\mathrm{P}^{\mathrm{Lm}}{ }_{\max }$ is the rated capacity of line-m, $\mathrm{n}$ is the exponent and wm a real non-negative weighting coefficient which may be used to reflect the importance of lines. PI will be small when all the lines are within their limits and reach a high value when there are overloads. Thus, it provides a good measure of severity of the line overloads for given state of the power system. Most of the works on contingency selection algorithms utilize the second order performance indices which, in general, suffer from masking effects. In this study, the value of exponent has been taken as 2 and $\mathrm{w}_{\mathrm{i}}=1$.

The real power flow PI sensitivity factors with respect to the parameters of TCSC can be defined as

$b_{k}=\left.\frac{\partial P I}{\partial x_{c k}}\right|_{x c k=0}$

The sensitivity of PI with respect to TCSC parameter connected between bus-i and bus-j can be written as 
$\frac{\partial P I}{\partial x_{c k}}=\sum_{m=1}^{N L} w_{m} P^{3}{ }_{L m}\left(\frac{1}{P^{\max }{ }_{L m}}\right)^{4} \frac{\partial P I}{\partial x_{c k}}$

With the sensitivity indices computed for TCSC, following criteria can be used for its optimal placement:

(a) In reactive power loss reduction method, TCSC should be placed in a line having the most positive loss sensitivity index.

(b) In PI method, TCSC should be placed in a line having most negative sensitivity index.

\section{RESULTS AND DISCUSSIONS}

To find the optimal locations of TCSC, the analysis has been implemented on IEEE 14 bus system which is shown in the Fig. 2. MATPOWER, a toolbox of MATLAB, has been used for simulations [11]. One of the approach solvers for OPF in MATPOWER is based on linear programming.

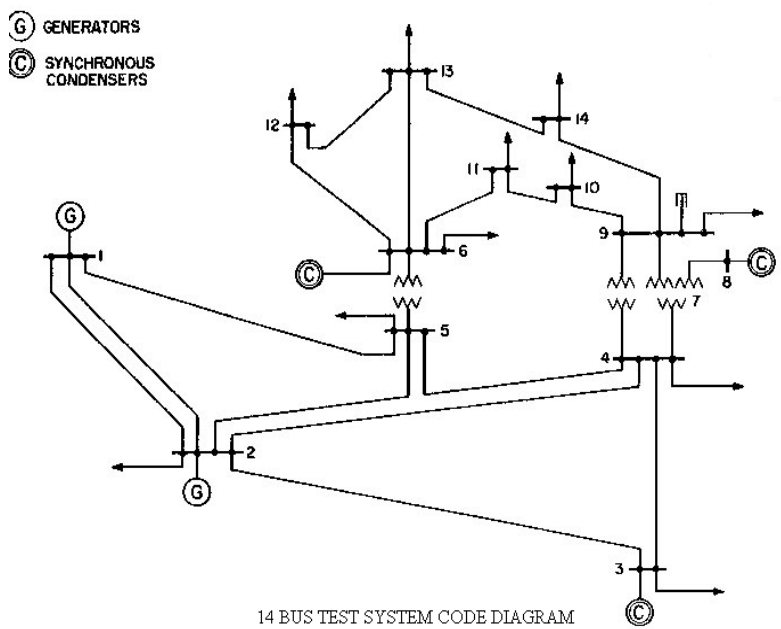

Fig.2. IEEE 14 bus System

The sensitivities of reactive power loss reduction and real power flow performance index with respect to TCSC control parameter has been computed and are shown in Table II

Table I

Power Flow of IEEE 14 Bus Systems

\begin{tabular}{|c|c|c|c|c|c|}
\hline Line & $\mathrm{i}-\mathrm{j}$ & $\begin{array}{c}\text { Power } \\
\text { Flow(pu) }\end{array}$ & Line & $\mathrm{i}-\mathrm{j}$ & $\begin{array}{c}\text { Power } \\
\text { Flow(pu) }\end{array}$ \\
\hline 1 & $5-6$ & 0.29574 & 11 & $7-8$ & 0.09671 \\
\hline 2 & $4-7$ & 0.16015 & 12 & $7-9$ & 0.11670 \\
\hline 3 & $4-9$ & 0.08574 & 13 & $9-10$ & 0.10296 \\
\hline 4 & $1-2$ & 0.04685 & 14 & $6-11$ & 0.10896 \\
\hline 5 & $2-3$ & $\mathbf{1 . 1 4 6 5 0}$ & 15 & $6-12$ & 0.26028 \\
\hline 6 & $2-4$ & 0.20876 & 16 & $6-13$ & 0.16028 \\
\hline 7 & $1-5$ & 0.10876 & 17 & $9-14$ & 0.31308 \\
\hline 8 & $2-5$ & 0.08259 & 18 & $10-11$ & 0.00308 \\
\hline 9 & $3-4$ & 0.12956 & 19 & $12-13$ & 0.02811 \\
\hline 10 & $4-5$ & 0.54423 & 20 & $13-14$ & 0.09651 \\
\hline
\end{tabular}

. The sensitive line in each case is presented in bold type. It can be observed from Table II that placement of TCSC in line-5 is suitable for reducing the total reactive power loss. System power flow result after placing TCSC in line-5 is shown in Table III. The value of control parameter of TCSC for computing power flow is taken as $\mathbf{0 . 9 8 8 5} \mathbf{p u}$.

Table II

Calculated Sensitivity Indices

\begin{tabular}{|l|c|c|c|c|c|}
\hline Line & $\mathrm{a}_{\mathrm{ij}}$ & $\mathrm{b}_{\mathrm{ij}}$ & Line & $\mathrm{a}_{\mathrm{ij}}$ & $\mathrm{b}_{\mathrm{ij}}$ \\
\hline
\end{tabular}

\begin{tabular}{|l|l|l|l|l|l|}
\hline 1 & -0.415 & $\mathbf{- 0 . 0 0 1}$ & 11 & -0.682 & -0.0335 \\
\hline 2 & -0.146 & -0.114 & 12 & -0.505 & -0.1808 \\
\hline 3 & -0.208 & -0.046 & 13 & $\mathbf{- 0 . 0 3 6}$ & -0.097 \\
\hline 4 & -0.208 & -0.068 & $\mathbf{1 4}$ & $\mathbf{- 0 . 0 3 2 5}$ & -0.025 \\
\hline 5 & $\mathbf{- 0 . 0 8 6}$ & -0.064 & 15 & $\mathbf{- 0 . 0 8 5}$ & -0.782 \\
\hline 6 & -0.108 & -0.0712 & 16 & -0.769 & -0.369 \\
\hline 7 & $\mathbf{- 0 . 0 4 4}$ & $\mathbf{- 0 . 0 0 0 3}$ & 17 & -0.049 & -0.457 \\
\hline 8 & -0.091 & -0.3714 & 18 & -0.803 & -0.0521 \\
\hline 9 & -0.116 & -0.350 & 19 & -0.622 & -0.114 \\
\hline 10 & -0.102 & $\mathbf{- 0 . 0 0 4}$ & 20 & -0.554 & -0.0336 \\
\hline
\end{tabular}

Table III

Power Flow of IEEE 14 Bus System after placing TCSC in line 5

\begin{tabular}{|c|c|c|c|c|c|}
\hline Line & $\mathrm{i}-\mathrm{j}$ & $\begin{array}{c}\text { Power } \\
\text { Flow(pu) }\end{array}$ & Line & $\mathrm{i}-\mathrm{j}$ & $\begin{array}{c}\text { Power } \\
\text { Flow(pu) }\end{array}$ \\
\hline 1 & $5-6$ & 0.29574 & 11 & $7-8$ & 0.09671 \\
\hline 2 & $4-7$ & 0.16635 & 12 & $7-9$ & 0.11670 \\
\hline 3 & $4-9$ & 0.08574 & 13 & $9-10$ & 0.12296 \\
\hline 4 & $1-2$ & 0.04685 & 14 & $6-11$ & 0.13696 \\
\hline 5 & $2-3$ & $\mathbf{0 . 9 1 4 5 2}$ & 15 & $6-12$ & 0.26028 \\
\hline 6 & $2-4$ & 0.23476 & 16 & $6-13$ & 0.16028 \\
\hline 7 & $1-5$ & 0.10876 & 17 & $9-14$ & 0.31308 \\
\hline 8 & $2-5$ & 0.08259 & 18 & $10-11$ & 0.00308 \\
\hline 9 & $3-4$ & 0.12956 & 19 & $12-13$ & 0.02811 \\
\hline 10 & $4-5$ & 0.54423 & 20 & $13-14$ & 0.09651 \\
\hline
\end{tabular}

It can be observed from Table III that congestion has been relieved. Placement of TCSC in line-1 also will reduce the total system reactive power loss but it will be less effective than placing a TCSC in line- 5 as can be seen from its sensitivity factors. It can be observed from Table II that placing a TCSC in line-5 is optimal for reducing the PI and congestion relief. System power flow result after placing TCSC in line-7 is shown in Table IV. The value of control parameter of TCSC for computing power flow is taken as $\mathbf{0 . 0 4 2 3}$ pu. It can be observed from Table IV that congestion has been relieved. Placement of TCSC on line-5 will reduce the PI value but it will be less effective than placing a TCSC in line-7 as can be seen from its sensitivity factors.

Table IV

Power Flow of IEEE 14 Bus System after placing TCSC in line 7

\begin{tabular}{|c|c|l|c|c|c|}
\hline Line & $i-j$ & $\begin{array}{c}\text { Power } \\
\text { Flow(pu) }\end{array}$ & Line & $i-j$ & $\begin{array}{c}\text { Power } \\
\text { Flow(pu) }\end{array}$ \\
\hline 1 & $5-6$ & 0.25740 & 11 & $7-8$ & 0.09171 \\
\hline 2 & $4-7$ & 0.12015 & 12 & $7-9$ & 0.10670 \\
\hline 3 & $4-9$ & 0.07574 & 13 & $9-10$ & 0.10296 \\
\hline 4 & $1-2$ & 0.04605 & 14 & $6-11$ & 0.18960 \\
\hline 5 & $2-3$ & $\mathbf{0 . 9 4 6 5 0}$ & 15 & $6-12$ & 0.26028 \\
\hline 6 & $2-4$ & 0.28761 & 16 & $6-13$ & 0.16028 \\
\hline 7 & $1-5$ & 0.11580 & 17 & $9-14$ & 0.31308 \\
\hline 8 & $2-5$ & 0.08259 & 18 & $10-11$ & 0.00308 \\
\hline 9 & $3-4$ & 0.12956 & 19 & $12-13$ & 0.02110 \\
\hline 10 & $4-5$ & 0.54423 & 20 & $13-14$ & 0.09510 \\
\hline
\end{tabular}

Total costs of two methods are $4678.9 \$$ and $\mathbf{5 0 4 0 . 5 1 \$}$. It can be observed that reduction of total system VAR power loss method is more economical than PI method for placing the TCSC and congestion management.

Single Line Outage as a Contingency Analysis: In a power system, if a line is corrupted, its power flow will be shared among other lines of the system. This will lead to possible overloading of some of the lines. Among 20 lines in 
IEEE 14 bus System, we selected 5 more important lines (line 1, 2, 3, 4 and 5) that have larger line outage sensitivity factors as candidates for placement of TCSC.

Table V

Line Outage Sensitivity Factors for IEEE 14 Bus System for Outage of line 1

\begin{tabular}{|c|c|c|c|c|c|}
\hline Line & $\mathrm{i}-\mathrm{j}$ & $\mathrm{d}_{1, \mathrm{k}}$ Factors & Line & $\mathrm{i}-\mathrm{j}$ & $\begin{array}{c}\mathrm{d}_{1, \mathrm{k}} \\
\text { Factors }\end{array}$ \\
\hline 1 & $5-6$ & - & 11 & $7-8$ & 0.045 \\
\hline 2 & $4-7$ & -0.425 & 12 & $7-9$ & 0.00399 \\
\hline 3 & $4-9$ & -0.125 & 13 & $9-10$ & 0.0128 \\
\hline 4 & $1-2$ & -0.265 & 14 & $6-11$ & 0.00065 \\
\hline 5 & $2-3$ & -0.255 & 15 & $6-12$ & -0.0369 \\
\hline 6 & $2-4$ & -0.0201 & 16 & $6-13$ & -0.0447 \\
\hline 7 & $1-5$ & -0.325 & 17 & $9-14$ & -0.0364 \\
\hline 8 & $2-5$ & -0.0299 & 18 & $10-11$ & -0.0458 \\
\hline 9 & $3-4$ & -0.0214 & 19 & $12-13$ & 0.00369 \\
\hline 10 & $4-5$ & 0.0569 & 20 & $13-14$ & 0.0369 \\
\hline
\end{tabular}

The effects of line outage on this network are studied here. By opening each of the lines of the system, we consider the effect of opened line on remaining of the system. If there is still congestion in the network, then we try to set the installed TCSC in such a way that congestion is relived. If congestion still persists in the system, we shall install a new TCSC by using reactive power loss reduction method or open the congested line/s.

Table VI

Line Outage Sensitivity Factors for IEEE 14 Bus System for Outage of line 2

\begin{tabular}{|c|c|c|c|c|c|}
\hline Line & $\mathrm{i}-\mathrm{j}$ & $\mathrm{d}_{\mathrm{l}, \mathrm{k}}$ Factors & Line & $\mathrm{i}-\mathrm{j}$ & $\begin{array}{c}\mathrm{d}_{1, \mathrm{k}} \\
\text { Factors }\end{array}$ \\
\hline 1 & $5-6$ & 0.025 & 11 & $7-8$ & 0.0189 \\
\hline 2 & $4-7$ & - & 12 & $7-9$ & 0.00169 \\
\hline 3 & $4-9$ & 0.214 & 13 & $9-10$ & 0.0065 \\
\hline 4 & $1-2$ & 0.296 & 14 & $6-11$ & 0.0006 \\
\hline 5 & $2-3$ & 0.1254 & 15 & $6-12$ & -0.0139 \\
\hline 6 & $2-4$ & -.0144 & 16 & $6-13$ & -0.0015 \\
\hline 7 & $1-5$ & -0.1362 & 17 & $9-14$ & -0.0156 \\
\hline 8 & $2-5$ & -.00158 & 18 & $10-11$ & -0.0250 \\
\hline 9 & $3-4$ & -0.0124 & 19 & $12-13$ & 0.0024 \\
\hline 10 & $4-5$ & 0.0367 & 20 & $13-14$ & 0.0164 \\
\hline
\end{tabular}

Outage of line 5-6: System power flow by opening line 5-6 is shown in Table V. From Table V, it is found that by opening line 5-6, $\mathrm{d}_{1, \mathrm{k}}$ Factors has been calculated and line 3-5 has been congested. Now if we set the control parameter of TCSC, xc, in line 3-5 pu to $\mathbf{0 . 5 2 8 3}$ pu then power flow of line $1-4$ will be $0.99956 \mathrm{pu}$ and congestion will be relieved. Therefore, by setting the installed TCSC in line 3-5 congestion has been relieved. Outage of line 4-7: System power flow by opening line 4-7is shown in Table VI. From this table, it can be observed that by opening line 4-7, line 7-8 has been congested. Now if we set the control parameter of TCSC, $x_{c}$, in line 3-5 to $\mathbf{0 . 7 7 4 0 4 5}$ pu then power flow of line $2-5$ will be $\mathbf{0 . 9 9 9 5 6}$ pu and congestion will be relieved.

\section{CONCLUSION}

Congestion management is an important issue in deregulated power systems. FACTS devices such as TCSC by controlling the power flows in the network can help to reduce the flows in heavily loaded lines. Because of the considerable costs of FACTS devices, it is important to obtain optimal location for placement of these devices. The results presented in this paper show that sensitivity index along with TCSC cost should be effectively used for determining optimal location of TCSC. The effect of TCSC on line outage in order to relieve congestion has also been studied. It can be observed from the results of line outage that we can relieve congestion by setting the installed TCSC.

\section{REFERENCES}

[1] D. Shirmohammadi, B. Wollenberg, A. Vojdani, P. Sandrin, M. Pereira,F. Rahimi, T. Schneider, and B. Stott, "Transmission dispatch and congestion management in the emerging energy market structures," IEEE Trans. Power Syst., vol. 13, pp. 1466-1474, Nov. 1998.

[2] H. Y. Yamin and S. M. Shahidehpour, "Transmission congestion and voltage profile management coordination in competitive electricity markets," International Journal of Electrical Power \& Energy Systems, vol. 25, pp. 849-861, Dec. 2003.

[3] M. I. Alomoush and S. M. Shahidehpour, "Contingencyconstrained congestion management with a minimum number of adjustments in preferred schedules," International Journal of Electrical Power \& Energy Systems, vol. 22, pp. 277-290, May 2000.

[4] S. Bruno, M. L. Scala, R. Sbrizzai, and G. Vimercati, "Replicating interruptible supply contracts for security constrained transmission management," in Proc. IEEE Power Tech, vol. 4, pp. 558-564, June 2003.

[5] S. N. Singh and A. K. David, "Optimal location of FACTS devices for congestion management," Electr. Power Syst. Res., vol. 58, pp. 71-79, June 2001.

[6] P. Preedavichit and S. C. Srivastava, "Optimal reactive power dispatch considering FACTS devices," Electr. Power Syst. Res., vol. 46, pp. 251-257, Sep.1998.

[7] Naresh Acharya and N. Mithulananthan, "Locating series FACTS devices for congestion management in deregulated electricity markets," Electric power Systems Research, vol. 77, pp. 352-360, March 2007.

[8] G. B. Shrestha and Wang Feng, "Effects of series compensation on spot price power markets," Electric Power Systems Research, vol. 27, pp. 428-436, March 2005.

[9] S.N. Singh and A. K. David, "Optimal location of FACTS devices for congestion management," Electric Power Systems Research, vol. 58, pp. 71-79, Oct. 2000.

[10] A. J. Wood and B. E. Wollenberg, Power generation, operation and control, 2nd ed., New York: Wiley Interscience, 1996.

[11] MATPOWER, a MATLAB power system simulation package. Version 3.0.0. http://www.pserc.cornell.edu/ matpower. 